\title{
Incoherent exciton trapping in self-similar aperiodic lattices
}

\author{
Francisco Domínguez-Adame and Enrique Maciá* \\ Departamento de Física de Materiales, Facultad de Físicas, Universidad Complutense, E-28040 Madrid, Spain
}

\begin{abstract}
Angel Sánchez
Theoretical Division and Center for Nonlinear Studies, Los Alamos National Laboratory, Los Alamos, New Mexico 87545 and Escuela Politécnica Superior, Universidad Carlos III de Madrid, C./ Butarque 15, E-28911 Leganés, Madrid, Spain
\end{abstract}

(Received 22 July 1994; revised manuscript received 12 September 1994)

\begin{abstract}
Incoherent exciton dynamics in one-dimensional perfect lattices with traps at sites arranged according to aperiodic deterministic sequences is studied. We focus our attention on Thue-Morse and Fibonacci systems as canonical examples of self-similar aperiodic systems. Solving numerically the corresponding master equation we evaluate the survival probability and the mean-square displacement of an exciton initially created at a single site. Results are compared to systems of the same size with the same concentration of traps randomly as well as periodically distributed over the whole lattice. Excitons progressively extend over the lattice on increasing time and, in this sense, they act as a probe of the particular arrangements of traps in each system considered. The analysis of the characteristic features of their time decay indicates that exciton dynamics in self-similar aperiodic arrangements of traps is quite close to that observed in periodic ones, but differs significantly from that corresponding to random lattices. We also report on characteristic features of exciton motion suggesting that Fibonacci and Thue-Morse orderings might be clearly observed by appropriate experimental measurements. In the conclusions we comment on the implications of our work on the way towards a unified theory of the ordering of matter.
\end{abstract}

\section{INTRODUCTION}

Interest in the study of the physical properties of elementary excitations in one-dimensional (1D) self-similar aperiodic systems has considerably grown during the last years. Albeit these systems were originally considered as somewhat intermediate between the periodic (crystalline) and random (amorphous, glassy) orderings of matter, it has been progressively realized that systems containing basic units arranged according to the Fibonacci, ${ }^{1-3}$ Thue-Morse, ${ }^{4,5}$ period-doubling, ${ }^{6}$ or Rudin-Shapiro ${ }^{7}$ sequences display novel properties which are not shared by the systems usually considered in condensed matter physics. In this way, we have recently provided strong evidence supporting the idea that selfsimilar aperiodic systems reveal a new kind of order, namely, aperiodic order, rather than representing a confuse mixture of periodic order and randomness. ${ }^{8,9}$ As experimental realizations of such systems become available in the fields of quasicrystalline phase research ${ }^{10}$ and multilayered heterostructures technology, ${ }^{11-15}$ interest in these aperiodically ordered forms of matter goes beyond a mere conceptual interest.

It is well known that self-similar aperiodic systems, described by tight-binding and Kroning-Penney models, possess singular continuous energy spectra. This point has been rigorously proven for Fibonacci, ${ }^{16,17}$ perioddoubling, and Thue-Morse ${ }^{18}$ sequences and it has recently been conjectured that this spectral type may be a common characteristic of all aperiodic systems obtained by the application of a substitution sequence ${ }^{19}$ Accord- ingly, numerical analyses have shown that this kind of spectra exhibits a highly fragmented structure, with a hierarchy of splitting subbands displaying self-similar patterns and that the associated (generalized) eigenstates behave in a very peculiar manner, characterized by dramatic spatial fluctuations and becoming neither localized nor extended in the usual sense. ${ }^{20-23}$ Hence, the question as to whether the peculiar structure of the energy spectrum of self-similar aperiodic systems influences the transport properties through the sample follows in a natural way. ${ }^{8}$ In this work we will investigate incoherent exciton dynamics in $1 \mathrm{D}$ self-similar aperiodic systems, considering the Fibonacci and Thue-Morse sequences as canonical examples. The aim of this study is threefold. In the first place we show how time evolution of quasiparticles (excitons in the present case) may be usefully employed to determine structural features of lattices. In particular, we demonstrate that excitons, initially created at a single site, act as a probe of the underlying structure as time evolves and the quasiparticle interacts with larger and larger regions of the system via the combined action of diffusion and trapping. In this sense exciton dynamics might be regarded as a diagnostic tool from an experimental viewpoint. In the second place we ascertain how self-similar order modifies exciton dynamics in comparison with the dynamics associated with the longrange disorder of random systems. Finally, we determine the differences between exciton propagation through periodic chains and exciton transport in aperiodic systems displaying quasiperiodic order (Fibonacci) on the one hand, and nonquasiperiodic order (Thue-Morse) on the other hand. In this way we are able to report on two 
interesting results. First, regarding exciton dynamics, self-similar aperiodic lattices are more similar to periodic lattices than they are to random ones and, second, exciton motion in quasiperiodic lattices also differs from the corresponding motion in nonquasiperiodic chains.

We will report on these issues according to the following scheme. In Sec. II we describe our model and the physical magnitudes we will compute in order to properly characterize exciton dynamics. Section III contains our main results concerning survival probabilities and meansquare displacements of incoherent excitons, along with the corresponding interpretation of the obtained results. Section IV concludes the paper with a brief account on practical implications of our results.

\section{MODEL}

We consider excitations in a 1D lattice whose time evolution is described by the following master equation for the probability $P_{k}(t)$ to find the exciton at site $k:{ }^{24}$

$$
\frac{d}{d t} P_{k}=W\left(P_{k+1}+P_{k-1}-2 P_{k}\right)-G_{k} P_{k},
$$

where $W>0$ is the intersite rate constant, which is assumed to be independent of $k$ hereafter and $G_{k}$ is the trapping rate at site $k$. The quantity of interest in luminescence experiments is the survival probability $n(t)$ defined as

$$
n(t)=\sum_{k} P_{k}(t)
$$

where the index $k$ runs over all lattice sites. Moreover, assuming that the excitation is initially at site $k_{0}$ $\left[P_{k}(0)=\delta_{k k_{0}}\right]$, we can also calculate the mean-square displacement of the excitation (which is related to the diffusion coefficient ${ }^{24}$ ) as follows:

$$
R^{2}(t)=\sum_{k}\left(k-k_{0}\right)^{2} P_{k}(t),
$$

where the lattice spacing is taken to be unity hereafter. These two functions characterize the dynamics of excitons in the lattice under the combined action of diffusion and trapping. Thus, it is known that, in infinite lattices without traps $\left(G_{k}=0\right)$, the survival probability is conserved $(n(t)=1)$ and the mean-square displacement increases linearly with time $\mathrm{e}^{25}\left[R^{2}(t)=2 D t, D\right.$ being the diffusion coefficient].

In what follows we consider that $G_{k}$ can only take on two values $G_{A}=0$ and $G_{B}=G>0$; that is, only sites $B$ are able to trap excitons. We will arrange sites $A$ and $B$ according to the Thue-Morse sequence and the Fibonacci sequence, at random, or periodically, depending on the particular kind of lattice we are interested in. For convenience we define $c$ as the ratio between the number of traps and the total number of sites in the considered lattice, $N$. Deterministic aperiodic sequences can be generated by simple substitution rules. Thus, we have $A \rightarrow A B, B \rightarrow B A$ for the Thue-Morse sequence and $A \rightarrow A B, B \rightarrow A$ for the Fibonacci one. Finite, self- similar lattices are obtained in this way by $l$ successive applications of the substitution rule. The $l$ th generation lattice has $2^{l}$ elements for the Thue-Morse lattice (TML) and $F_{l}$ elements for the Fibonacci lattice (FL), where $F_{l}$ denotes the Fibonacci numbers. Such numbers are generated from the recurrence relationship $F_{l}=F_{l-1}+F_{l-2}$ with $F_{0}=F_{1}=1$; as $l$ increases the ratio $F_{l-1} / F_{l}$ converges toward $\tau=(\sqrt{5}-1) / 2=0.618 \ldots$, which is known as the inverse golden mean. Therefore, sites are arranged according to the sequence $A B B A B A A B \ldots$ in the TML and $A B A A B A B A \ldots$ in the FL. The value of $c$ is strictly equal to 0.5 for any generation of the TML. On the contrary, the value of $c$ depends on the particular generation of the FL, but for large enough systems one has $c \sim 1-\tau=0.3819 \ldots$. Disordered lattices are obtained by placing traps (sites $B$ ) at random over the lattice, maintaining fixed the concentration of traps c. Finally, we consider in this work periodic lattices of two types. One of them is set with $c=0.5$ and traps placed at sites with even index. This periodic lattice will be compared to the TML. The other type is obtained from a periodic superposition of unit cells of the form $A B A A B A B A$, which is nothing but the fifth-order approximant to the Fibonacci sequence. The concentration of traps is $c=0.375$ for this periodic arrangement, a value rather close to the value $1-\tau$ corresponding to infinite FL's.

\section{NUMERICAL RESULTS AND DISCUSSION}

We have numerically solved the master equation (1) for Thue-Morse, random, and periodic lattices of $N=$ $2^{10}=1024$ units and for Fibonacci, random, and periodic lattices of $N=F_{15}=987$ units using an implicit (Crank-Nicholson) integration scheme. To avoid free ends effects, spatial periodic boundary conditions are introduced, so that the detailed balance required by Eq. (1) is preserved. The initial condition for the exciton motion is $P_{k}(0)=\delta_{k k_{0}}$, with $k_{0}=500\left(k_{0}=494\right)$ for lattices with $N=1024(N=987)$ sites; that is, we will assume that the exciton is created, for instance, by a pulsed excitation, roughly at the middle of the lattice. The trapping rate $G$ will be measured in units of $W$ whereas time will be expressed in units of $W^{-1}$. The maximum integration time and the integration step are 250 and $5 \times 10^{-4}$, respectively. Smaller time steps led to similar results. Since we are mainly interested in the effects due to particular arrangements of traps rather than in a detailed description of the influence that the different parameters have in the incoherent motion of excitations, we will fix the values of $W$ and $G$ henceafter. Thus we have set $W=1$ and $G=0.2$ as representative values. For disordered lattices a series of random distributions of traps was generated for a given trap concentration, and ensembles comprising a number of realizations varying from 50 to 200 were averaged to check the convergence of the computed mean values. Since convergence was always satisfactory between all the ensembles, we present the results corresponding to 50 averages. 
The obtained results for the mean-square displacement and survival probability of excitons propagating through the TML are presented in Figs. 1(a) and 1(b), respectively, along with the corresponding results for random and periodic lattices with a trap concentration of $c=0.5$. Analogous magnitudes describing the motion of incoherent excitons through the FL and related random and periodic lattices are shown in Figs. 2(a) and 2(b). Let us consider, in the first place, the behavior of the meansquare displacement of incoherent excitons through these systems. In all cases it becomes apparent that the time evolution of $R^{2}(t)$ arises from the competition between two different processes, namely, diffusion (the exciton is transferred from site to site, starting at $k_{0}$ ) and trapping (the exciton progressively decays in time since possible detrapping processes are not considered in our model). At short times the first mechanism dominates because the exciton is still close to the initial position and, consequently, there exist small chances to be trapped. As time elapses, the trapping mechanism plays a major role since the exciton can be found in a larger segment of the lattice. This competition gives rise to the occurrence of a well-defined maximum in $R^{2}(t)$, whose position de-
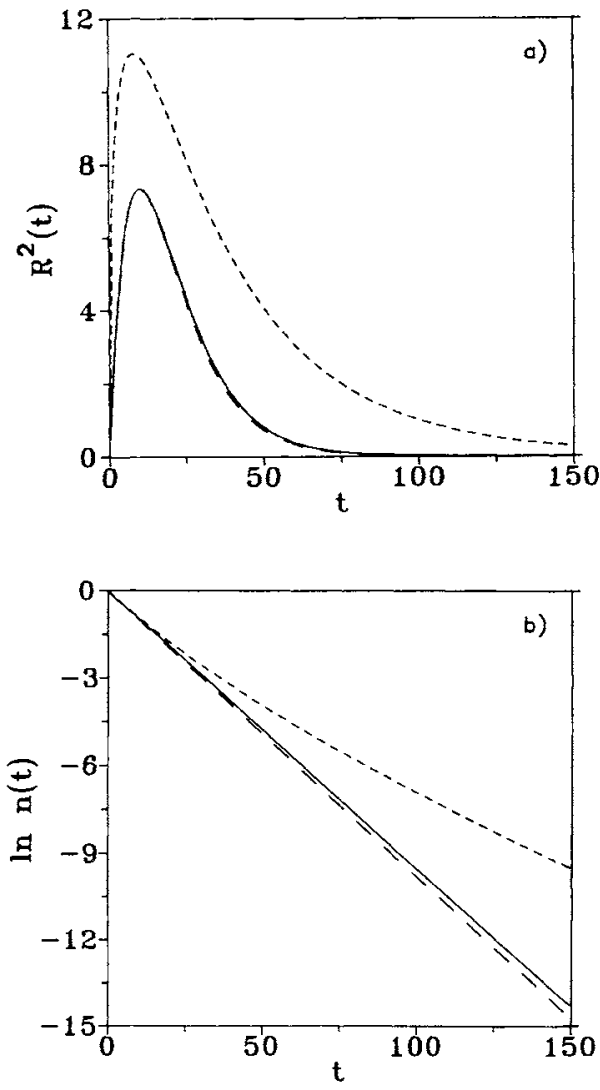

FIG. 1. (a) Mean-square displacement and (b) logarithm of the survival probability of excitons as a function of time for lattices of $N=1024$ sites with $c=0.5$. Results correspond to Thue-Morse (solid lines), periodic (long-dashed lines), and random (short-dashed lines) arrangements of traps.
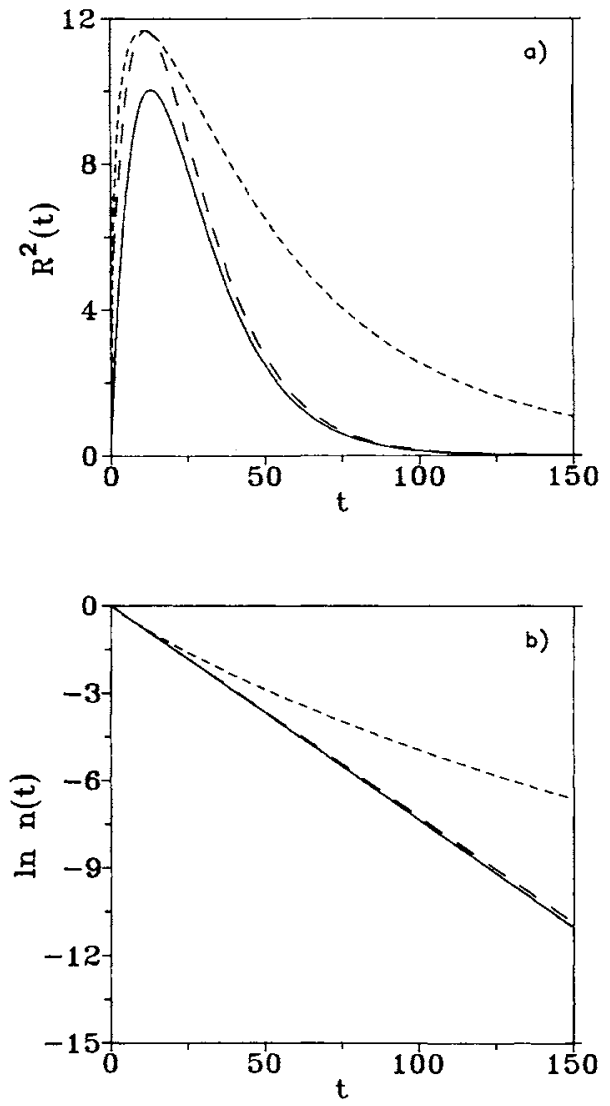

FIG. 2. (a) Mean-square displacement and (b) logarithm of the survival probability of excitons as a function of time for lattices of $N=987$ sites with $c=0.382$. Results correspond to Fibonacci (solid lines), periodic (long-dashed lines), and random (short-dashed lines) arrangements of traps.

pends not only on the concentration of traps but also on the spatial distribution of these traps. In addition to this quite general behavior we observe significant differences between the exciton behavior in quasiperiodic (Fibonacci) and nonquasiperiodic (Thue-Morse) aperiodic lattices. In fact, the mean-square displacement of an exciton propagating through a TML essentially coincides with that corresponding to the case of the periodic lattice over the entire time interval we have considered. Moreover, the $R^{2}(t)$ curve describing the exciton motion in the random lattice appreciably differs from both the TML and periodic corresponding curves. On the contrary, the mean-square displacement of excitons in the FL cannot be easily compared with that of excitons moving in neither periodic nor random lattices at short times but, as time increases, exciton motion in FL's progressively resembles that taking place in the periodic lattice approximant.

Now, we turn our attention to the evolution of the survival probability. It is well known that, for any periodic distribution of traps, the behavior of the survival probability is simply exponential in time, and is given by the expression $n(t)=\exp (-c G t)$, whereas in random 
lattices it presents a more complex and nonexponential dependence on time. Keeping this fact in mind, the interpretation of Figs. 1(b) and 2(b) is straightforward. The rate of trapping of incoherent excitons in both the TML and FL is very similar to that of the corresponding periodic lattices with the same fraction of traps and quite different from that associated with the corresponding random lattices. 'Therefore, from this point of view, self-similar aperiodic systems behave as periodic ones in a very close manner. In particular we note that not only an exponential decay rate for both kind of aperiodic systems is observed, but the slope of the corresponding survival probabilities fits the value prescribed by the trap concentration $c$ appearing in the general expression for periodic systems. Finally, note that the decay rate in random lattices is much slower than in the other lattices (periodic and aperiodic).

\section{CONCLUSIONS}

From the comparison of the mean-square displacement and survival probability plots for the lattices considered in this work, several conclusions can be drawn. In the first place we point out that excitons propagating through self-similar aperiodic lattices behave in a very similar way as they do in periodic $1 \mathrm{D}$ systems, and consequently exhibit a time evolution completely different from that they show in random systems. A second important result emerging from our numerical simulations is that the exciton dynamics in the TML significantly differs from that recorded in the FL at short times. This can be easily seen by comparing the corresponding mean-square displacement curves. The justification for this effect can be accounted for starting from the following picture: As time evolves the exciton progressively extends over the lattice and, in this sense, it acts as a probe, indicating the rate of trapping associated with the particular arrangement of traps of the underlying structure. In this way, the shape of the $R^{2}(t)$ curve can be interpreted in a topological sense. As has been explained previously the characteristic maximum of this curve indicates a cutoff between two different transport regimes in the system. At short times we have classical diffusion through the lattice; meanwhile at longer times the effects of trapping become dominant. Figure 1(a) indicates that excitons propagate through the TML as they will do through a periodic lattice having the same trap concentration in both regimes. Hence, nonquasiperiodic order associated with the Thue-Morse sequence has no relevant effects on exciton dynamics and, as long as transport properties are concerned, Thue-Morse and binary periodic arrangements with a trap concentration $c=0.5$ are completely equivalent. On the contrary, the shape of the $R^{2}(t)$ curve, shown in Fig. 2(a), clearly reveals that quasiperiodic order has a profound effect on the excitonic diffusion transport regime. In fact, we see that diffusion of excitons in the FL is considerably lower than that taking place in both periodic and random lattices at the same times. This result, along with the fact that the area determined from the expression $\int_{0}^{\infty} R^{2}(t) d t$ is also smaller than the corresponding values for the other lattices, led us to the conclusion that trapping processes are more efficient for quasiperiodic arrangements than they are for other possible orderings, including both periodic (crystalline) and random (glassy) structures. This interesting result may be of relevance from an experimental point of view, since by properly measuring the value of diffusion constants in aperiodic lattices, we should be able to estimate the kind of underlying topological order they present.

The results reported on in this work provide substantial support to the view that certain classes of aperiodic systems, like those arranged according to the Fibonacci and Thue-Morse sequences, are notable representatives of new orderings of matter, displaying a higher level of order than usual periodic systems are able to do. ${ }^{9}$ This view might be further elaborated by introducing the concept of hierarchies of order. ${ }^{26} \mathrm{By}$ this we mean that rather than thinking of different kinds of order, classified into separated categories ranging from those more periodic to those more random, as has been the usual procedure, it may be more fruitful to separate different kinds of order according to a well-established criteria such as quasiparticle behavior and transport properties.

\section{ACKNOWLEDGMENTS}

This work is partially supported by Universidad Complutense through Project No. PR161/93-4811. A.S. is partially supported by DGICyT (Spain) Grant No. PB92-0248, by MEC (Spain)/Fulbright, and by the European Union Network ERBCHRXCT930413. Work at Los Alamos is performed under the auspices of the U.S. DOE.
* Also at Instituto de Estudios Interdisciplinares, El Guijo, Z4 Galapagar, E-28260 Madrid, Spain.

${ }^{1}$ M. Kohmoto and J. R. Banavar, Phys. Rev. B 34, 563 (1986).

2 S. Das Sarma and X. C. Xie, Phys. Rev. B 37, 1097 (1988).

${ }^{3}$ A. Chakrabarti, S. N. Karmakar, and R. K. Moitra, J. Phys. Condens. Matter 1, 1017 (1989).

${ }^{4}$ R. Riklund and M. Severin, J. Phys. C 21, L965 (1988).

${ }^{5}$ S. Tamura and F. Nori, Phys. Rev. B 40, 9790 (1989); 41,
$7941(1990)$

${ }^{6}$ D. Barache and J. M. Luck, Phys. Rev. B 49, 15004 (1994).

${ }^{7}$ M. Dulea, M. Johansson, and R. Riklund, Phys. Rev. B 45, 105 (1992); 46, 3296 (1992); 47, 8547 (1993).

${ }^{8}$ E. Maciá, F. Domínguez-Adame, and A. Sánchez, Phys. Rev. B 49, 9503 (1994).

${ }^{9}$ E. Maciá, F. Domínguez-Adame, and A. Sánchez, Phys. Rev. E 50, 679 (1994).

${ }^{10}$ L. X. He, X. Z. Li, Z. Zhang, and K. H. Kuo, Phys. Rev. 
Lett. 61, 1116 (1988).

${ }^{11}$ R. Merlin, K. Bajema, R. Clarke, F. Y. Yuang, and P. K. Bhattacharya, Phys. Rev. Lett. 55, 1768 (1985).

${ }^{12}$ R. Merlin, K. Bajema, J. Nagle, and K. Ploog, J. Phys. (Paris) Colloq. 48, C5-503 (1987).

${ }^{13}$ G. Carlotti, D. Fioretto, L. Palmieri, G. Socino, L. Verdini, H. Xia, A. Hu, and X. K. Zhang, Phys. Rev. B 46, 12777 (1992).

14 J. Birch, M. Severin, U. Wahlström, Y. Yamamoto, R. Radnoczi, R. Riklund, and L. R. Wallenberg, Phys. Rev. B 41, 10938 (1990).

${ }^{1.5}$ J. L. Cohn, J. J. Lin, F. J. Lamelas, H. He, R. Clarke, and C. Uher, Phys. Rev. B 38, 2326 (1988).

${ }^{16}$ A. Sütö, Commun. Math. Phys. 111, 409 (1987); J. Stat. Phys. 56, 525 (1989).

${ }^{17}$ J. Bellisard, B. Iochum, E. Scoppola, and D. Testard, Commun. Math. Phys. 125, 527 (1989).
${ }^{18}$ J. Bellissard, A. Bovier, and J. -M. Ghez, Commun. Math. Phys. 135, 379 (1991).

${ }^{19}$ A. Bovier and J.-M. Ghez, Commun. Math. Phys. 158, 45 (1993).

${ }^{20} \mathrm{M}$. Severin and R. Riklund, Phys. Rev. B 39, 10362 (1989).

${ }^{21}$ Y. Kim, M. H. Lee, and M. Y. Choi, Phys. Rev. B 40, 2581 (1989).

${ }^{22}$ G. Y. Oh, C. S. Ryu, and M. H. Lee, J. Phys. Condens. Matter 4, 8187 (1992).

${ }^{23}$ J. X. Zhong, J. R. Yan, and J. Q. You, J. Phys. Condens. Matter 3, 5685 (1991).

${ }^{24}$ S. Alexander, J. Bernasconi, W. R. Schneider, and R. Orbach, Rev. Mod. Phys. 53, 175 (1981).

${ }^{25}$ R. Silbey, in Disordered Solids. Structures and Processes (Plenum Press, New York, 1989).

${ }^{26}$ E. Maciá and F. Domínguez-Adame, Phys. Rev. B 50, 16856 (1994). 